\title{
X-ray luminosity functions of different morphological and X-ray type AGN populations
}

\author{
M. Pović ${ }^{1,2}$, $^{\star}$ A. M. Pérez García ${ }^{1,3}$, M. Sánchez-Portal ${ }^{5,3}$, A. Bongiovanni ${ }^{1,4,3}$, J. Cepa ${ }^{4,1}$, M. \\ Fernández Lorenzo $^{1,2}$, M. A. Lara-López ${ }^{1,6}$, J. Gallego ${ }^{7}$, A. Ederoclite ${ }^{1,8}$, I. Márquez ${ }^{2}$, J. Masegosa ${ }^{2}$, \\ E. Alfaro ${ }^{2}$, H. Castañeda ${ }^{9}$, J. I. González-Serrano ${ }^{10}$, and J. J. González ${ }^{11}$ \\ 1 Instituto de Astrofísica de Canarias (IAC), La Laguna, Tenerife, Spain \\ 2 Instituto de Astrofísica de Andalucía (IAA-CSIC), Granada, Spain \\ 3 Asociación ASPID, Apartado de Correos 412, La Laguna, Tenerife, Spain \\ 4 Departamento de Astrofísica, Universidad de La Laguna (ULL), La Laguna, Spain \\ ${ }^{5}$ Herschel Science Centre, ESAC/INSA, Madrid, Spain \\ 6 Australian Astronomical Observatory (AAO), Sidney, Australia \\ 7 Departamento de Astrofísica y CC. de la Atmósfera, Universidad Complutense de Madrid, Madrid, Spain \\ 8 Centro de Estudios de Fsica del Cosmos de Aragón (CEFCA), Teruel, Spain \\ 9 Escuela Superior de Física y Matemáticas, Intituto Politécnico Nacional (IPN), Mexico D.F, Mexico \\ 10 Instituto de Física de Cantabria, CSIC-Universidad de Cantabria, Santander, Spain \\ 11 Instituto de Astronomía UNAM, México D.F, México
}

Received ?, accepted?

Published online later

Key words galaxies: active - galaxies: luminosity function $-\mathrm{X}$-rays: galaxies

Luminosity functions are one of the most important observational clues when studying galaxy evolution over cosmic time. In this paper we present the X-ray luminosity functions for X-ray detected AGN in the SXDS and GWS fields. The limiting fluxes of our samples are $9.0 \times 10^{-15}$ and $4.8 \times 10^{-16} \mathrm{erg} \mathrm{cm}^{-2} \mathrm{~s}^{-1}$ in the $0.5-7.0 \mathrm{keV}$ band in the two fields, respectively. We carried out analysis in three $\mathrm{X}$-ray bands and in two redshift intervals up to $\mathrm{z} \leq 1.4$. Moreover, we derive the luminosity functions for different optical morphologies and X-ray types. We confirm strong luminosity evolution in all three bands, finding the most luminous objects at higher redshift. However, no signs of density evolution are found in any tested X-ray band. We obtain similar results for compact and early-type objects. Finally, we observe the 'Steffen effect', where $\mathrm{X}$-ray type-1 sources are more numerous at higher luminosities in comparison with type-2 sources.

(c) ?? WILEY-VCH Verlag GmbH \& Co. KGaA, Weinheim

\section{Introduction}

The luminosity function (LF) is among the fundamental tools for studying the formation and evolution of galaxies (e.g. Schechter 1976). Galaxies with an Active Galactic Nucleus (AGN) in their centres are among the most luminous sources in the Universe. Therefore, they provide unique information about the properties of high-redshift galaxies that is important for understanding how galaxies formed and evolved. However, the manner in which a galaxy enters the active phase, the duration of the activity, and the origin of the accreting material have not been clarified yet. The use of LFs to determine the distribution and evolution of the AGN accretion history has been shown to be essential for constraining models of formation and evolution of supermassive black holes (SMBHs), AGN feedback, fuelling mechanisms, and the relation between active and non-active galaxies (e.g., Aird et al. 2010, La Franca et al. 2010, Fiore et al. 2012 and references therein). LF computation requires

^ Corresponding author: e-mail: mpovic@iaa.es the availability of data for large samples of sources, covering a wide range of redshift and luminosity, in order to accurately determine the shape of the LF and its evolution over cosmic time.

$\mathrm{X}$-ray emission is one of the principal characteristics of AGN activity. Since X-ray data are relatively unaffected by absorption, they have been shown to be very useful for selecting large AGN samples. Hard and very-hard X-ray surveys are especially important, since they are able to detect significant populations of sources that are obscured in optical and NIR bands. Therefore, X-ray data provide the most complete and unbiased samples of AGN, and the X-ray luminosity function (XLF) can provide important knowledge about AGN evolution over cosmic time (e.g., Barger et al. 2005; Barger \& Hasinger et al. 2005).

The AGN LF has been widely studied over past years, providing important constraints on models of galaxy formation and evolution. In the last decade, deep X-ray (e.g., Ueda et al. 2003; La Franca et al. 2005; Hopkins, Richards, \& Hernquist 2007; Silverman et al. 2008a, 2008b; Ebrero et al. 2009; Yencho et al. 2009; Aird et al. 2010 and references therein), optical (e.g., Boyle et al. 2000; Fan et al. 2001, 
2004; Croom et al. 2004; Richards et al. 2005, 2006; Jiang et al. 2006; Cool et al. 2012), IR (Dai et al. 2009 and references therein; Wu et al. 2011 and references therein) and radio (e.g., Kaiser \& Best 2007; Kellermann et al. 2012 and references therein) surveys studied large samples of AGN over a broad range of redshift $(\mathrm{z} \lesssim 6)$. These multiwavelength studies have provided a wider view of AGN properties. As already mentioned, $\mathrm{X}$-ray selection provides one of the most complete samples of active galaxies; however they may miss the most obscured (Compton thick) sources. These sources can be observed in the IR. In contrast, optical data are important for providing the redshift and AGN morphology. Current observations show that the

AGN space density reaches a broad peak at $\mathrm{z} \approx 1-3$ (e.g. Hasinger et al. 2005; Silverman et al. 2008b; Aird et al. 2008; Brusa et al. 2009) followed by a decline of $\approx 2 \mathrm{dex}$ to the present day (e.g., Ueda et al. 2003; Hasinger et al. 2005; Barger et al. 2005). Moreover, X-ray studies (e.g., Page et al. 1997; Miyaji et al. 2000, 2001; La Franca et al. 2002; Cowie et al. 2003; Ueda et al. 2003; Hasinger et al. 2005; Aird et al. 2010 and references therein), but also optical, IR, and radio measurements (e.g., Hunt et al. 2004; Cirasuolo, Magliocchetti, \& Celotti 2005; Matute et al. 2006) indicate that the space density of low-luminosity AGN peaks at lower redshift than the bright AGN. In order to explain this effect, called 'cosmic downsizing' (Cowie et al. 2003; Merloni 2004; Heckman et al. 2004, Babić et al. 2007; Fanidakis et al. 2012), most of the proposed models of AGN formation and evolution consider different forms of AGN feedback processes as the responsible mechanism (Di Matteo et al. 2005; Hopkins et al. 2006; Croton et al. 2006; Hopkins et al. 2008).

Many studies of the AGN LF have been published, leading to different models of AGN evolution. However, their analyses were restricted to the evolution of the entire AGN population.In this work, in addition to a study of the entire AGN sample, the X-ray luminosity function was also studied for different morphologies and X-ray obscurations for redshifts $\mathrm{z} \leq 1.4$. The results presented here represent a pilot study of a much larger sample of AGN that will be carried out in the COSMOS field (Scoville et al. 2007) with the final aim of studying the evolution of different AGN triggering mechanisms over cosmic time. The paper is structured as follows: in Section 2, we describe the data used in this work, including X-ray (Section 2.1), optical (Section 2.2), near- and mid-infrared (NIR and MIR) data (Section 2.3. and provide brief summary of photometric redshift and kcorrection estimations of the optical counterparts of X-ray emitters (Section 2.4). In Section 3, we describe the optical morphology and X-ray type classification of X-ray selected AGN in both fields. In Section 4 we present the analysis of the XLFs, with the luminosity function measurements described in Section 4.1 and discussion of the LFs of the whole AGN sample in Section 4.2, of the different morphological types in Section 4.3, and of the different X-ray types in Section 4.4 Our study of the evolution of X-ray type-1 sour-ces is given in Section 4.5. Finally, the results obtained in this work are summarized in Section 5 .

The concordance cosmology with $\Omega_{\Lambda}=0.7, \Omega_{M}=0.3$, and $\mathrm{H}_{0}=70 \mathrm{~km} \mathrm{~s}^{-1} \mathrm{Mpc}^{-1}$ is assumed. Unless otherwise specified, all magnitudes are given in the $\mathrm{AB}$ system (Oke \& Gunn 1983).

\section{Observational data}

This work involves sources belonging to two fields: $\mathrm{Su}-$ baru/XMM-Newton Deep Survey (SXDS) and Groth-Westphal Strip (GWS). These fields were selected for the OSIRIS Tunable Emission Line Object (OTELO) survey (Cepa et al. 2008). This is a deep on-going survey of emission line sources that are being observed with tunable filters in the Optical System for Imaging and low Resolution Integrated Spectroscopy (OSIRIS) on the Gran Telescopio de Canarias (GTC1) telescope. Results presented in this paper complement studies of the AGN/QSO LFs carried out in the OTELO survey also using OSIRIS data.

The SXDS is a large survey covering $>1$ square degree centred at RA $=02^{h} 18^{m}$ and DEC $=-05^{\circ} 00^{\prime}$ ' (Sekiguchi et al. in prep), and observed from $\mathrm{X}$-ray to radio.

The GWS field is a part of the Extended Groth Strip (EGS) centred at RA $=14^{h} 17^{m}$ and DEC $=+52^{\circ} 30^{\prime}$. Broad band optical $B V R I$ observations were performed by the OTELO group (Cepa et al. 2008). The EGS was also observed by the All-Wavelength Extended Groth Strip International Survey (AEGIS2) team, with the aim of obtaining deep images and spectroscopic data from X-ray to radio, covering an area of 0.5 - 1 square degree (Davis et al. 2007).

We mainly use X-ray and optical data in this work. NIR and MIR data were used to complement the optical information for the estimation of photometric redshifts estimations (see Section 2.4). The observational data are briefly described below.

\subsection{X-ray data}

The SXDS field was observed with XMM-Newton at seven pointings in the $0.2-10 \mathrm{keV}$ energy range (PI Michael G. Watson) and covering $\simeq 1.3 \mathrm{deg}^{2}$. The central observation is deepest with a nominal exposure time of $\sim 100 \mathrm{ksec}$. It is surrounded by six shallower observations each with an exposure of $\sim 50 \mathrm{ksec}$.

The required European Photon Imaging Camera (EP$\left.\mathrm{IC}^{3}\right)$ data were collected from the XMM-Newton v.5.0 scientific archive (XSA 4 ) and were processed by our team using standard procedures of the Science Analysis System (SAS5) v7.1.2 package, and the latest relevant Current Calibration Files (CCF). Source detection was based on the

\footnotetext{
1 http://www.gtc.iac.es/

${ }^{2}$ http://aegis.ucolick.org/

$3 \mathrm{http}: / / \mathrm{xmm}$.esac.esa.int/external/xmm_user_support/documentation /technical/EPIC/

${ }^{4} \mathrm{http} / / / \mathrm{xmm}$.esac.esa.int/xsa/

5 http://xmm.esac.esa.int/external/xmm_data_analaysis
} 
edetect chain SAS procedure. In order to minimize spurious detections the likelihood threshold parametert was set at $\mathrm{L}=14$. Thus the probability that the source is real exceeds $\mathrm{P}=0.99$ which implies less than one fake source per instrument, per pointing, and per band. A detailed description of X-ray data reduction and source detection is available in Pović et al. (2012). We detected 1121 sources with fluxes greater than our flux detection limit of $9.0 \times 10^{-15}$ erg $\mathrm{cm}^{-2} \mathrm{~s}^{-1}$ in the $0.5-7.0 \mathrm{keV}$ band.

The GWS field was observed with Chandra covering three continuous fields centred at the original HST GWS. All observations were carried out by the AEGIS collaboration using the Advanced CCD Imaging Spectrometer (ACIS-17) instrument, with a total exposure time of $\sim 200 \mathrm{ksec}$ in each field, covering an area of $0.08 \mathrm{deg}^{2}$.

All datasets were gathered from the Chandra Data Archive 8 and were processed by our team using the Chandra Interactive Analysis of Observations (CIAO) 9 v4.0 package and Calibration Data Base (CALDB) v3.4.0. Standard reduction procedures were applied to produce new level 2 event files, restricted to the $0.5-8 \mathrm{keV}$ range in order to avoid high background spectral regions. Good time intervals were defined by means of celldetect and a threshold of $\mathrm{S} / \mathrm{N}=3$. Absolute astrometry was improved, where the average positional accuracy improvement is $6.3 \%$ with respect to the original astrometry provided by the spacecraft attitude files, and the event files were merged. We applied the CIAO wavdetect Mexican-Hat wavelet source detection program to all bands. We set a significance threshold of $2 \times 10^{-7}$, expecting about 12 spurious sources in the entire catalogue. The final catalogue has 639 unique $\mathrm{X}$-ray emitters, with fluxes greater than the flux detection limit of $4.8 \times 10^{-16} \mathrm{erg} \mathrm{cm}^{-2} \mathrm{~s}^{-1}$ in the $0.5-7.0 \mathrm{keV}$ band. A detailed description of $\mathrm{X}$-ray data reduction and source detection can be found in Pović et al. (2009).

In this paper, we derive the XLF in the $0.5-2 \mathrm{keV}$ (soft) and $0.5-7 \mathrm{keV}$ (total) bands for both the SXDS and GWS samples, and in the $2-10 \mathrm{keV}$ (hard) band for the SXDS sample. To separate between the X-ray unobscured and obscured sources (see Section 3), we use $\operatorname{HR}(2-4.5 \mathrm{keV} /$ $0.5-2 \mathrm{keV}$ ) hardness ratio defined as:

$$
H R=\frac{C R(2-4.5 k e V)-C R(0.5-2 k e V)}{C R(2-4.5 k e V)+C R(0.5-2 k e V)},
$$

where $\mathrm{CR}$ is the count rate in a given energy band.

We tested the reliability of our X-ray number counts in the SXDS and GWS fields by comparing with number counts obtained in deeper (e.g. Chandra Deep Field North

\footnotetext{
${ }^{6} \mathrm{~L}=-\ln (1-\mathrm{P})$, where $\mathrm{P}$ is the probability to have a spurious detection due to random Poisson fluctuations.

7 http://cxc.harvard.edu/cal/Acis/

8 http://asc.harvard.edu/cda/

9 http://cxc.harvard.edu/ciao/
}

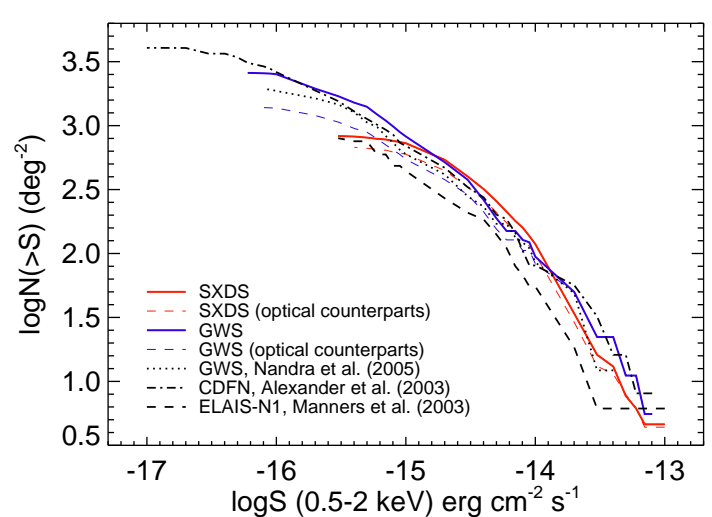

Fig. 1 Cumulative $\log N-\log S$ functions for the SXDS and GWS fields in the soft band, for all detected X-ray sources (red and blue thick solid lines, respectively) and for X-ray emitters with optical counterpart (red and blue thin dashed lines, respectively). Three distributions, with different exposure times and effective areas, are represented for comparison: GWS by Nandra et al. (2005) (black dotted line), CDFN (black dash-dot-dashed line), and ELAIS-N1 (black dashed line).

(CDFN); Alexander et al. 2003) and less deep (e.g., ELAISN1; Manners et al. 2003) surveys. Figure 1 shows the cumulative number count distributions $(\log N-\log S)$ per square degree in the soft band. Two curves have been computed for the SXDS and GWS sample, one for the complete sample of X-ray detections and the other for the sample of Xray detections with optical counterparts. For objects brighter than $\sim 4 \times 10^{-14} \mathrm{erg} \mathrm{s}^{-1} \mathrm{~cm}^{-2}$ in the case of SXDS, and $\sim 10^{-14} \mathrm{erg} \mathrm{s}^{-1} \mathrm{~cm}^{-2}$ in the case of GWS, both distributions (complete $\mathrm{X}$-ray sample and $\mathrm{X}$-ray sample with optical counterparts) coincide, while for fainter objects the density of X-ray sources with optical counterparts starts to decrease. In contrast, the difference in the density of X-ray sources between the distributions of all objects and optical counterparts is lower in the case of the SXDS sample, since the optical data are deeper in comparison with the GWS sample. Since X-ray data are deeper in the GWS field, and since it is important to deal with the complete sample when measuring the LF, we will use the limiting fluxes corresponding to the completeness levels of SXDS samples in all analyses.

\subsection{Optical data}

The SXDS optical imaging observations were carried out with the Suprime-Cam on the Subaru Telescope We observed five continuous sub-fields covering an area of 1.2 $\mathrm{deg}^{2}$ with a total integration time of 133 hours (Furusawa et al. 2008). We downloaded and used publicly available catalogues from the SXDS web page 10 , obtaining photometric

\footnotetext{
${ }^{10}$ http://www.naoj.org/Science/SubaruProject/SXDS/index.html
} 
information in five broad-band filters $B, V, R_{c}, i^{\prime}$, and $z$, with limiting AB magnitudes of 28.4, 27.8, 27.7, 27.7, and 26.6 , respectively. The total number of objects in each field is around 900000.

GWS observations used in this work were carried out by the OTELO team using the Prime Focus Imaging Platform (PFIP) at the William-Herschel Telescope (WHT) 11 covering a total area of $0.18 \mathrm{deg}^{2}$. Three pointings were observed, each of them using the $B, V, R$, and $I$ broadband filters, and performing several exposures at each pointing of 600,800 , 900 , or $1000 \mathrm{sec}$ depending on the filter. Standard reduction procedures have been applied. Resulting limiting AB magnitudes are 24.8, 24.9, 24.6 and 23.8 in $B, V, R$, and $I$ band, respectively. The total number of objects is around 44000 . A detailed description of data reduction and optical source detection can be found in Cepa et al. (2008).

\subsection{NIR and MIR data}

NIR information for the SXDS field was taken from the UKIDSS DR2 catalogue12 (Hewett et al. 2006; Warren et al. 2007; Hodgkin et al. 2009). This data has AB limiting magnitudes of 23.4, 23.4, and 22.9 in $J, H$, and $K$ bands, respectively, and covers an area of $8.75 \mathrm{deg}^{2}$. MIR data for the SXDS field was obtained with the Spitzer telescope as part of the XMM-LSS survey which is one of the areas surveyed within the Spitzer Wide-area IR Extragalactic Legacy Survey (SWIRE) covering a total area of $9.1 \mathrm{deg}^{2}$. We used the public Infrared Array Camera (IRAC13) catalogue 14 of approximately 250700 objects, with $\mathrm{AB}$ limiting magnitudes of $21.0,21.1,19.5$, and 19.4 in the $3.6,4.5,5.8$, and $8.0 \mu \mathrm{m}$ bands, respectively.

We used publicly available NIR $K_{s}$ Palomar data 15 for the GWS field. The catalogue contains $\sim 45000$ objects with an AB limiting magnitude of 20.6 covering an area of $0.7 \mathrm{deg}^{2}$. Spitzer/IRAC EGS catalogue 16 is publicly available and has been used in this work to gather MIR data for GWS X-ray emitters. The raw catalogues contain (57.4, $48.0,16.3,13.6) \times 10^{3}$ objects, with $\mathrm{AB}$ limiting magnitudes of 23.2, 22.8, 21.3, and 21.1 in the four IRAC channels, respectively, and a total area of 0.33 square degrees.

\subsection{Redshifts and k-corrections of optical counterparts}

We cross-matched the optical data with X-ray catalogues using a search radius of $3^{\prime \prime}$ and $2^{\prime \prime}$ for SXDS and GWS fields, respectively. Applying the de Ruiter methodology (de Ruiter et al 1977; Pović et al. 2009) we obtain a completeness of $99.9 \%$ and $98.3 \%$, and a reliability of $76.2 \%$ and

\footnotetext{
11 http://www.ing.iac.es/Astronomy/telescopes/wht/

12 http://www.ukidss.org/archive/archive.html

13 http://www.cfa.harvard.edu/irac/

$14 \mathrm{http}: / /$ swire.ipac.caltech.edu/swire/astronomers/data_access.html

15 http://www.astro.caltech.edu/AEGIS/

16 http://www.cfa.harvard.edu/irac/egs/
}

$87.3 \%$ in the SXDS and GWS fields, respectively. Due to the poor reliability in the SXDS field and, in order to decrease the number of spurious detections, we also applied the Sutherland \& Saunders (1992) methodology, measuring the reliability for all possible matches inside the selected radius, and finally selecting, as an optical counterpart, the object with the highest probability. This additional step drops the percent of possible fake identifications from $24 \%$ to $6 \%$, in cases where multiple matches are detected with similar probabilities. We find 808 and $340 \mathrm{X}$-ray sources with optical counterpart in the SXDS and GWS fields, respectively. The final covered areas are $1.2 \mathrm{deg}^{2}$ and $0.08 \mathrm{deg}^{2}$ in the SXDS and GWS fields, respectively. These are the areas that will be used for solid angle estimations in Section 4 and cover the overlap area of all the data employed to identify $\mathrm{X}$-ray sources and their optical counterparts, and to measure photometric redshifts.

We used the ZEBRA (Feldmann et al. 2006) code to compute photometric redshifts for optical counterparts. Due to the small number of previously available spectroscopic redshifts in the SXDS field (fifteen objects), and the lack of NIR data in the GWS survey (only K data available), photometric redshifts were also derived using the Hyper $Z$ code (Bolzonella et al. 2000) as an additional check. We implemented and used the templates from the SWIRE17 $\mathrm{li}^{-}$ brary (Polletta et al. 2007), including galaxies belonging to different morphological types, starbursts, different types of Seyfert galaxies, and type-1 and type-2 QSO templates. Comparing the results from ZEBRA and Hyperz, we accepted only sources where the redshift results from both codes agreed within less than 0.1 for $\mathrm{z}<1$ and 0.2 for $\mathrm{z} \geq 1$. Following this criterion we derived photometric redshifts for $308(38 \%)$ and $203(60 \%)$ objects in the SXDS and GWS fields respectively. We focus our studies on the 208 (SXDS) and 169 (GWS) objects that have redshifts below 1.4 in order to derive an LF for sources with reliable redshift estimates. Additionally, we compared our photometric redshift in the SXDS field with the spectroscopic values recently provided by the UDS team 18 . The relation is presented in Figure 2 (top panel). $73 \%$ and $86 \%$ of all objects have photometric-spectroscopic redshift differences below 0.1 and 0.15 , respectively. In the GWS field the comparison between photometric and used spectroscopic values is presented in Figure 2(bottom panel). In this field, 66\% and $81 \%$ of all objects show differences below 0.1 and 0.15 , respectively. In the following analysis, we used the spectroscopic redshifts when available, and otherwise our photometric estimates (69\% and $83 \%$ for samples from the SXDS and GWS fields, respectively).

The code kcorrect (Blanton et al. 2007) was used in both fields in order to obtain k-corrections for the optical fluxes. In contrast. X-ray fluxes were k-corrected by assuming a standard power law spectral energy distribution (SED) with $\Gamma=1.8$. No intrinsic absorption correction has

\footnotetext{
17 http://www.iasf-milano.inaf.it/ polletta/templates/swire_templates.html

18 http://www.nottingham.ac.uk/astronomy/UDS/data/dr3.html
} 

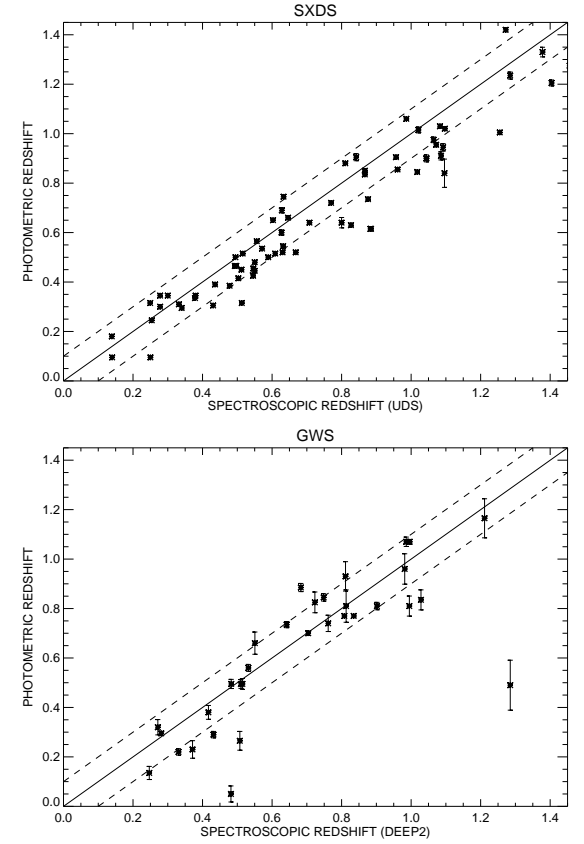

Fig. 2 Relation between our photometric and spectroscopic redshifts in the SXDS (top) and GWS (bottom) fields. Solid line shows the best correlation, when zphot=zspec, while the dashed lines show the \pm 0.1 difference limits.

been applied to the power law SED for objects considered to be unobscured(see Section 3) a fixed intrinsic absorption of $\mathrm{N}_{\mathrm{H}}=1.0 \times 10^{22} \mathrm{~cm}^{-2}$ was applied (e.g., Silverman et al. 2005; Younes et al. 2012) for obscured sources.

\section{Morphological and X-ray classification}

We used SExtractor (Bertin \& Arnouts 1996) and galSVM (Huertas-Company et al. 2008, 2009) codes to evaluate I-band morphologies for optical counterparts of $\mathrm{X}$ ray emitters in the SXDS field. SExtractor was used for detection of compact sources (CLASS_STAR $\geq 0.9$ ), while we classified extended objects with galSVM. As an additional check we made a visual classification for the largest and brightest objects using the IRAF / imexam (Tody 1986, 1993) tool and isophotal contour diagrams. The final classifications include $22 \%$ compact, $30 \%$ early-type, $18 \%$ latetype, $23 \%$ poorly classified and $7 \%$ unidentified sources. Further details about morphological classifications in the SXDS field can be found in Pović et al. (2012).

We obtained morphologies for X-ray emitters with optical counterparts in the GWS field using SExtractor (Bertin \& Arnouts 1996; to select compact sources as in the SXDS field) and GIM2D (Simard et al. 1998) codes, and we performed again a visual inspection as an additional check. Morphology in the GWS field was determined prior to the galSVM code becoming publicly available. The final classifications were carried out in the $\mathrm{R}$ band (the I band was affected by fringing) and included: $42 \%$ compact, $21 \%$ earlytype galaxies, $27 \%$ late-type galaxies, and $10 \%$ unidenti- fied sources. The number of compact objects is significantly higher in this field probably due to the lower quality of optical data compared to the SXDS field. Further details related with morphological classification in the GWS field can be found in Pović et al. (2009).

A coarse nuclear type classification was performed in both fields, based on a diagnostic diagram relating the $\mathrm{X}$ ray-to-optical $(\mathrm{X} / \mathrm{O})$ flux ratio and the hardness ratio $\mathrm{HR}$ $(2-4.5 \mathrm{keV} / 0.5-2 \mathrm{keV})$. The $\mathrm{X} / \mathrm{O}$ ratio was computed as ratio of fluxes $F_{0.5-4.5 \mathrm{keV}} / F_{R}$, where the optical flux $F_{R}$ was derived from the SExtractor auto magnitudes in the $R$ band. This flux ratio is useful to efficiently separate between active and non-active/Compton-thick galaxies (Alexander et al. 2001; Fiore et al. 2003; Civano et al. 2007), while the hardness ratio $\mathrm{HR}(2-4.5 \mathrm{keV} / 0.5-2 \mathrm{keV})$, measured as in equation (1), is very sensitive to absorption, and thus capable of separating X-ray type-1 (unobscured or unabsorbed) from X-ray type-2 (obscured or absorbed) AGN (Mainieri et al. 2002; Della Ceca et al. 2004; Perola et al. 2004; Caccianiga et al. 2004; Dwelly et al. 2005; Hasinger et al 2008). Full details on the methodology applied are given in Pović et al. (2009). Following Fiore et al. (2003), all objects having $\mathrm{X} / \mathrm{O}>0.1$ and $\mathrm{HR}(2-4.5 \mathrm{keV} / 0.5-2 \mathrm{keV})<-0.35$ have been classified as $\mathrm{X}$-ray type-1 (unobscured), while objects with $\mathrm{X} / \mathrm{O}>0.1$ and $\mathrm{HR}(2-4.5 \mathrm{keV} / 0.5-2 \mathrm{keV})>-0.35$ have been classified as X-ray type-2 (obscured) AGN. Figure 3 shows the $\mathrm{X}$-ray type classification diagram including the host morphological information (compact, early- and late-type galaxies). A large fraction of objects (59.3\%) fall inside the region of X-ray type-1 AGN. The dashed-line box indicates the 'highest probability' region for finding $\mathrm{X}$ ray type-1 AGN (Della Ceca et al. 2004). 34.9\% of our sample (SXDS + GWS) fall in this box while only $11 \%$ fall in the lower region of the diagram (i.e. typical of stars, normal galaxies and Compton-thick AGN). No evidence of any relationship between $\mathrm{X}$-ray and morphological types was indicated since there was no clear separation of $\mathrm{X}$-ray properties based on morphological types. Nevertheless, it can be seen that the majority (more than $60 \%$ ) of objects identified as compact are placed in the $\mathrm{X}$-ray type-1 region.

\section{XLFs of different AGN populations}

\subsection{LF measurements}

The LF is one of the most important tools for studying the evolution of extragalactic sources. It is defined as the number of objects per unit comoving volume per unit luminosity interval, i.e.

$$
\Phi(L, z)=\frac{d^{2} N}{d V d L}(L, z),
$$

where $N$ is the number of objects of luminosity $L$ found in the comoving volume $V$ at redshift $z$. In calculating the LF, 


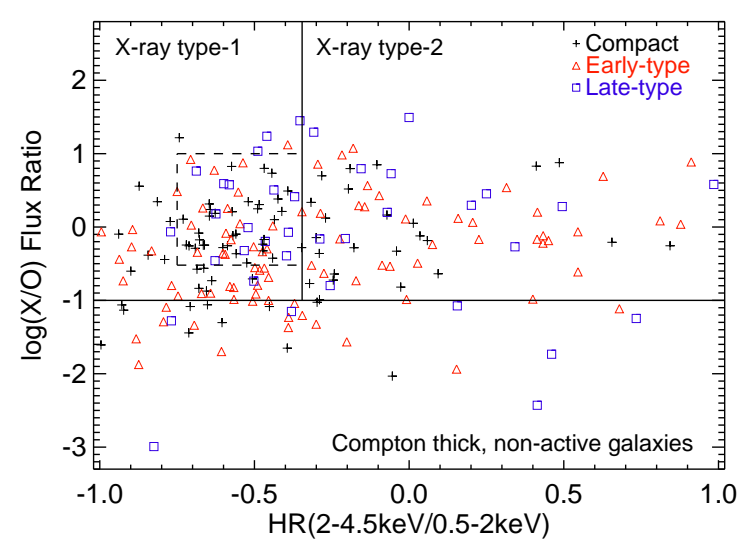

Fig. 3 Relation between the X/O flux ratio and $\mathrm{HR}(2-$ $4.5 \mathrm{keV}, 0.5-2 \mathrm{keV}$ ), for $\mathrm{X}$-ray emitters with optical counterparts in the SXDS and GWS fields. Sources belong to different morphological groups: compact (black crosses), early- (red triangles), and late-type (blue squares) galaxies. Solid lines separate $\mathrm{X}$-ray type-1 (unobscured) and X-ray type-2 (obscured) regions and the area with $\log \mathrm{X} / \mathrm{O}<-1$, where stars, non-active galaxies, and Compton thick AGN can be found (e.g. Fiore et al. 2003). The dashed line box presents the limits obtained by Della Ceca et al. (2004), presenting the 'highest probability' region for finding X-ray type-1 AGN.

we used the $1 / V_{a}$ method (Schmidt 1968) to measure the space density $(d N / d V)$ as:

$$
\frac{d N}{d V}(L, z) \approx \sum_{i=1}^{N} \frac{1}{V_{a}(i)},
$$

with $N$ objects having the luminosities and redshifts in the interval $\Delta L \Delta z$ around the centre of the bin $(L, z) . V_{a}(i)$ is the survey available comoving volume within which an object $i$ with luminosity $L(i)$ could be detected and remained in the redshift bin $\Delta z$. The main advantage of the $d N / d V$ estimator (compared with simply dividing the number of objects found by the average search volume) is that it takes into account the fact that more luminous objects are detectable over larger volumes than less luminous objects.

To derive the LF $(\Phi)$ through the space density in the observed luminosity bin and surveyed solid angle $(\Omega)$ we used the following equation:

$$
\Phi(\log L, z)=\frac{4 \pi}{\Omega} \frac{1}{\Delta \log L} \sum_{i=1}^{N} \frac{1}{V_{a}(i)} .
$$

A bin of logarithmic luminosity has been used in this work. The available comoving volume $V_{a}(i)$ has been measured through the comoving distance (e.g., Hogg 2000), for the concordance cosmology assumed in this work.

The statistical uncertainty of $\Phi$ was estimated using the following expression (e.g. Marshall 1985; Boyle, Shanks \& Peterson 1988; Page \& Carrera 2000):

$$
\delta \Phi=\frac{1}{\Delta L} \sqrt{\sum_{i=1}^{N}\left(\frac{1}{V_{a}(i)}\right)^{2}} .
$$

A Schechter (1976) function has been used to fit the LF:

$$
\Phi(L) d L=\Phi^{*}\left(\frac{L}{L^{*}}\right)^{\alpha} \exp \left(-\frac{L}{L^{*}}\right) \frac{d L}{L^{*}},
$$

where $\Phi(L) d L$ is the number of galaxies with luminosity between $L$ and $L+d L$ per $\mathrm{Mpc}^{3}$. This approximation has been widely used for more than 30 year and provides a good fit for both local (e.g. Loveday et al. 1992; Blanton et al. 2001) and high redshift sources (e.g. Norberg et al. 2002; Faber et al. 2007; Ouchi et al. 2008; Dai et al. 2009). The Schechter function has three free parameters which must be determined empirically: $\alpha, \Phi^{*}$ and $L^{*}$. The quantity $\alpha$ defines the faint end slope of the LF, and indicates how quickly galaxy number counts evolve at low luminosities. It is usually negative, implying a large number of galaxies with low luminosities; if $\alpha=-1$ the LF is said to be flat. $L^{*}$ is the so-called characteristic Schechter luminosity, and $\Phi^{*}$ is the number of galaxies per $\mathrm{Mpc}^{3}$ per luminosity at the characteristic luminosity.

We computed the best fit Schechter parameters using a Marquardt-Levenberg algorithm (Levenberg 1944; Marquardt 1963) by means of the IDL LMFIT 19 function, performing up to 100 iterations. Finally, the best fit parameters and their $1 \sigma$ uncertainty estimates, assuming Poisson statistics, were obtained.

\subsection{LF of whole AGN sample}

Our XLF analysis has been restricted to sources with $\mathrm{z} \leq 1.4$ in order to have a sample with reliable redshifts and an optimal number of sources in the $\mathrm{L}_{X}$-z plane (Figure 4). Figure 4 shows this plane in the total, and soft X-rays for AGN in both the SXDS and GWS fields, and in the hard band for the AGN belonging to the SXDS field only (since hard range was not selected in the GWS field). In both the soft and total bands, the objects with lowest X-ray luminosities belong to the GWS field, since the GWS Chandra data are deeper in comparison with the SXDS XMMNewton data (see Section 2.1). The diagrams show the Xray luminosity as a function of redshift for the entire source sample and for different morphological and X-ray types (from left to right). Luminosity limits in all three bands for both SXDS and GWS samples are also presented. In all bands we can clearly see the rapid increase of luminosity at lower redshifts, and flatter luminosity distribution at higher redshifts. Also, we can see that the most luminous objects are the most distant. At $0.7<\mathrm{z}_{2}<1.4$, we found more compact sources in the total and soft bands than in

\footnotetext{
${ }^{19}$ http://idlastro.gsfc.nasa.gov/idl_html_help/LMFIT.html\#wp831462
} 


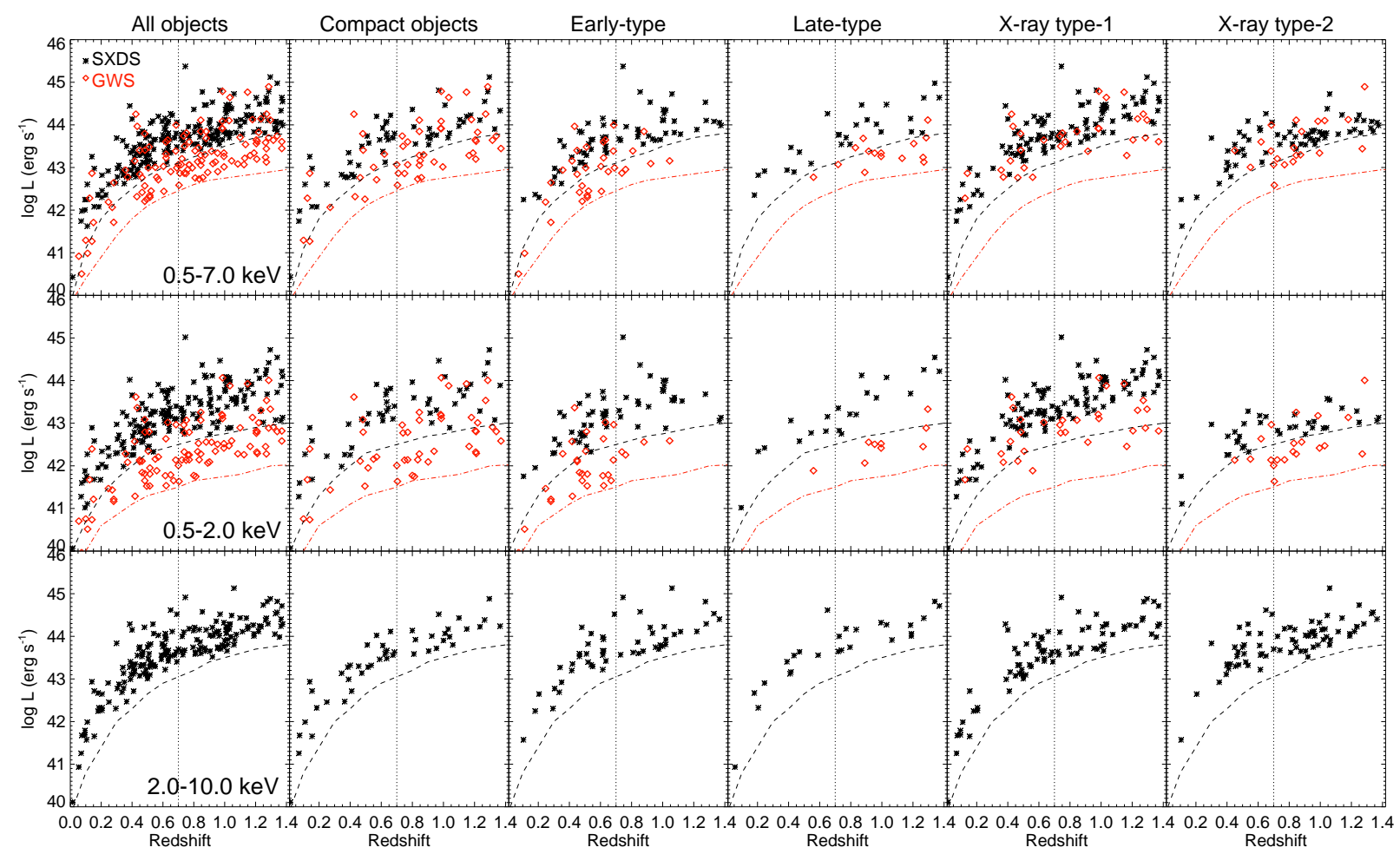

Fig. 4 From top to bottom: Relation between the X-ray luminosity in the $0.5-7.0 \mathrm{keV}, 0.5-2.0 \mathrm{keV}$, and $2.0-10.0 \mathrm{keV}$ bands and redshift for a sample of X-ray emitters with optical counterparts in the SXDS (black stars) and GWS (red diamonds) fields. From left to right: This relation is shown for the whole sample of objects, and for different morphological (compact, early- and late-type galaxies) and X-ray types (X-ray type-1 and type-2 sources). Vertical thin, dotted line defines the limit between two redshift intervals that we used to study the evolution in Sections 4.2 and 4.5. Black dashed and red dash-dot-dashed lines present the luminosity limits of the SXDS and GWS samples, respectively.

the hard X-rays. Early-type objects dominate late-types at lower redshifts in the total and soft bands, but have similar populations in the hard band.

XLFs derived in the $0.5-7.0 \mathrm{keV}$ (TXLF), $0.5-2.0 \mathrm{keV}$ (SXLF), and $2.0-10.0 \mathrm{keV}$ (HXLF) bands are presented in Figure 5 (left panel, from top to bottom, respectively). The LFs were analysed in two volumes with similar numbers of objects in all bands. The first volume corresponds to the redshift range $0<\mathrm{z}_{1} \leq 0.7$ that represents about $1 / 4$ of the total volume analysed $\left(\approx 0.009 \mathrm{Gpc}^{3}\right.$ for a covered solid angle), while the second volume corresponds to the redshift range $0.7<\mathrm{z}_{2} \leq 1.4$ occupying the rest of the volume. The TXLF and SXLF were derived from both SXDS and GWS samples to flux limits of $9.0 \times 10^{-15}$ and $2.25 \times 10^{-15} \mathrm{erg}$ $\mathrm{cm}^{-2} \mathrm{~s}^{-1}$ in each band, respectively. Since the X-ray GWS data are deeper than the SXDS data (see Section 2.1), the used limiting fluxes correspond to the completeness levels of the SXDS samples. The HXLF has been derived from the SXDS sample to a flux limit of $4.0 \times 10^{-15} \mathrm{erg} \mathrm{cm}^{-2} \mathrm{~s}^{-1}$. The distributions of objects belonging to each observed luminosity bin in the total, soft, and hard bands are shown in Figure 5(right panel, from top to bottom, respectively).
In order to estimate the error in the derivation of the LF due to cosmic variance when both the SXDS and GWS samples are analysed, the Cosmic Variance Calculator 20 (Trenti \& Stiavelli 2008) was used. Using both the Press-Schechter (Press \& Schechter 1974) and the Sheth-Tormen (Sh-eth \& Tormen 1999) models, the error in the LF due to cosmic variance is smaller than $10 \%$.

A good fit to the LF was obtained by using a Schechter function. The best fit Schechter parameters are given in Table 1 The shape of the LF remains similar in the three $\mathrm{X}-$ ray bands (in both redshift intervals). The faint-end slopes $(\alpha)$ of the luminosity function does not show a significant difference with redshift. While the SXLF shows similar behaviour in $\mathrm{z}_{1}$ and $\mathrm{z}_{2}$ bins, it shows a shallower faint-end slope $\alpha \sim-1.0$, suggesting slower evolution than the other two bands. The plots of Figure 5 taken together show that hard X-ray sources have higher luminosities than those selected in the $0.5-7.0 \mathrm{keV}$ band, a contrast even stronger in comparison with the soft $\mathrm{X}$-ray objects. This difference is also found in the luminosity density with soft $\mathrm{X}$-ray sources showing the lowest values.

\footnotetext{
20 http://casa.colorado.edu/ trenti/CosmicVariance.html
} 

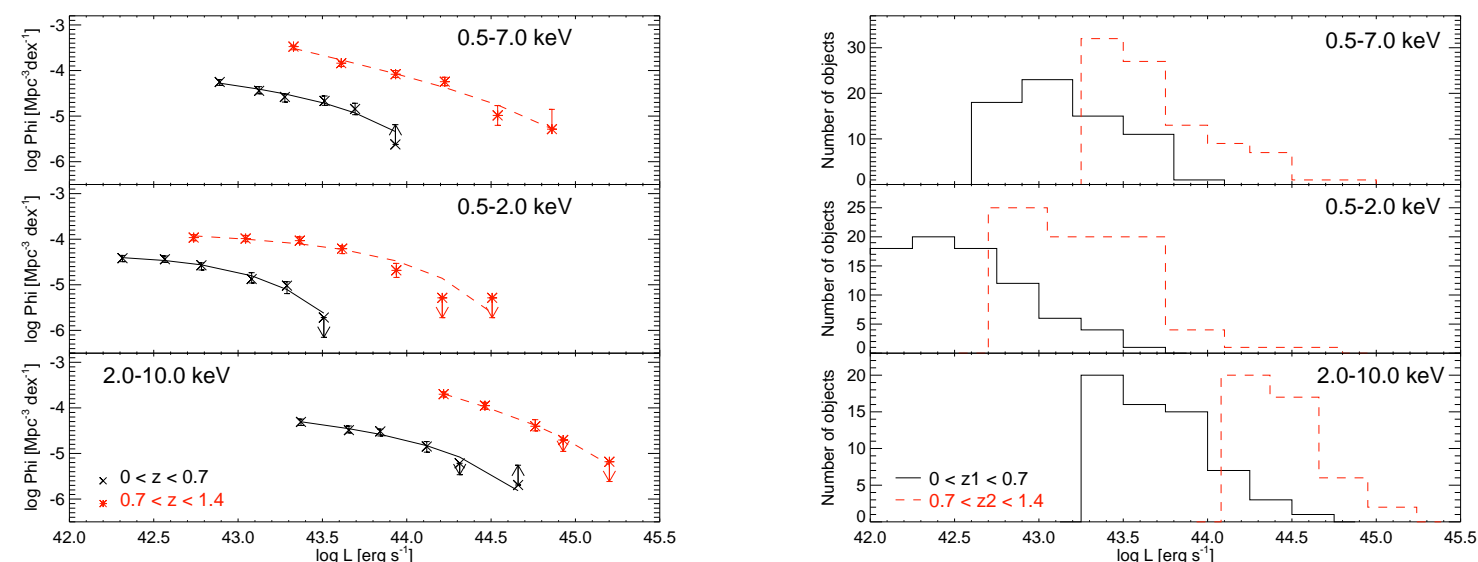

Fig. 5 (Left, from top to bottom): TXLF, SXLF, and HXLF in the $\mathrm{z}_{1} \leq 0.7$ (black solid line, $\mathrm{x}$ symbols) and $0.7 \leq \mathrm{z}_{2} \leq 1.4$ (red dashed line, stars) intervals. The best-fit Schechter parameters are shown in Table1 The arrows represent $1 \sigma$ errors in those luminosity bins where we have data only above or below the central luminosity. In all following figures, the arrows have the same significance. (Right, from top to bottom): Luminosity distribution in each redshift bin in the total, soft, and hard bands.

Table 1 The best-fit Schechter parameters of the XLF for a whole sample of AGN (Figure 5)

\begin{tabular}{c|ccccc}
\hline \hline $\begin{array}{c}\text { Energy range } \\
(\mathbf{k e V})\end{array}$ & $\mathbf{z}^{a}$ & $\mathbf{N u m}^{b}$ & $\begin{array}{c}\log \Phi^{*} \\
\left(\mathbf{M p c}^{-3}\right)\end{array}$ & $\alpha$ & $\begin{array}{c}\log L^{*} \\
\left(\mathbf{e r g ~ s}^{-1}\right)\end{array}$ \\
\hline \hline $\mathbf{0 . 5 - 7 . 0}$ & $0-0.7$ & 127 & $-4.49 \pm 0.66$ & $-1.35 \pm 0.57$ & $43.69 \pm 0.51$ \\
& $0.7-1.4$ & 126 & $-4.76 \pm 0.80$ & $-1.85 \pm 0.24$ & $44.82 \pm 0.59$ \\
\hline $\mathbf{0 . 5 - 2 . 0}$ & $0-0.7$ & 111 & $-4.31 \pm 0.31$ & $-0.99 \pm 0.44$ & $43.03 \pm 0.26$ \\
& $0.7-1.4$ & 99 & $-4.09 \pm 0.22$ & $-1.15 \pm 0.20$ & $43.98 \pm 0.18$ \\
\hline $\mathbf{2 . 0 - 1 0 . 0}$ & $0-0.7$ & 97 & $-4.55 \pm 0.42$ & $-1.35 \pm 0.38$ & $44.25 \pm 0.30$ \\
& $0.7-1.4$ & 87 & $-4.32 \pm 1.16$ & $-1.92 \pm 0.69$ & $44.98 \pm 0.63$ \\
\hline \hline
\end{tabular}

${ }^{a}$ Redshift range

${ }^{b}$ Number of objects

Analysing differences in evolution between $\mathrm{z}_{1}$ and $\mathrm{z}_{2}$ ranges we find:

\section{* Luminosity Evolution}

Strong XLF was found in all bands as shown in Figure 5 Sources typically show lower luminosities in $\mathrm{z}_{1}$ than in $\mathrm{z}_{2}$ band. The Schechter fits in the three X-ray energy ranges lead to the same conclusion: $L^{*}$ shows evolution with redshift, having lower values in $\mathrm{z}_{1}$ interval.

* Density Evolution

In general, there is no evidence for any significant AGN density evolution in any considered band. The sources become brighter as redshift increases, but given the uncertainties, their characteristic number density $\Phi^{*}$ associated to a characteristic luminosity $\left(L^{*}\right)$ is consistent between the two analysed redshift intervals.

Strong AGN luminosity evolution was also found in previous studies (e.g. Miyaji et al. 2001; Ueda et al. 2003; La Franca et al. 2005; Hasinger et al. 2005; Silverman et al. 2008a; Ebrero et al. 2009; Yencho et al. 2009; Aird et al.
2010 and references therein). This evolution shows that a larger population of high-luminosity AGN is found at higher redshifts, while low-luminosity AGN are dominant at lower redshifts. This could be a result of: (i) selection effects (e.g., as redshift increases, first, the detectability of low-luminosity AGN decreases, and second, the observed volume increases, thus increasing the probability of detecting a lar-ger number of high-luminosity sources), (ii) true evolutionary effects, or (iii) a mixture of both. Selection effects will be tested in future, using a much larger sample of active galaxies, however the most luminous sources we observe at high redshifts we do not observe in the local universe.

\subsection{LF of compact, early- and late-type active galaxies}

We also studied the AGN XLF in relation to morphology. The top panel in Figure 6 shows the XLF as a function of morphology, while the bottom panel shows the luminosity distribution for each morphological class. The LFs of compact, early- and late-type objects were derived over the full $0.5-7.0 \mathrm{keV}$ range. Best-fit Schechter parameters are 


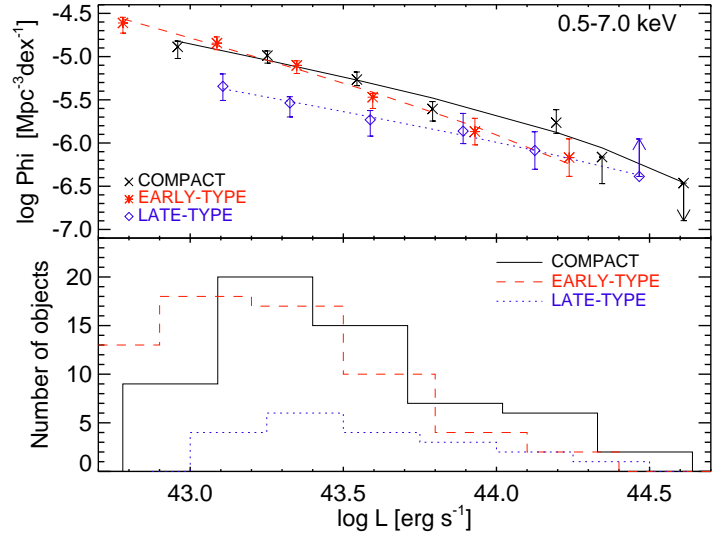

Fig. 6 (Top): TXLF derived for different morphological types: compact (black crosses), early- (red stars) and latetype (blue diamonds) sources in $\mathrm{z} \leq 1.4$ redshift interval. (Bottom): Distribution of objects in each luminosity bin of compact (black line), early- (red line) and late-type (blue line) objects. The best-fit Schechter parameters are shown in Table 2 .

given in Table 2. Due to the small number of objects in each morphological sample the LFs were derived without dividing into different redshift bins. Therefore, the evolution rates were not studied as a function of morphology in the framework of this paper. No significant differences were found within the uncertainties between LFs of compact and early-type objects in the total band. They have similar steep faint-end slopes and similar $L^{*}$ and $\Phi^{*}$ best-fit parameters. The situation may reverse for late-type objects showing a shallower faint-end slope, suggesting slower evolution, and higher break luminosity. However, a larger sample of latetype objects is necessary to confirm the results obtained.

These results are, in general, consistent with those of Georgakakis et al. (2009) who studied the contribution of different morphological types to the TXLF at redshifts 0.5 1.3 for the Extended Groth Strip field (EGS). Both this work and Georgakakis et al. (2009) found that relative to latetype objects, early-type hosts make a major contribution to the TXLF over the entire luminosity range, while at higher luminosities compact objects become more dominant.

\subsection{LF of X-ray type-1 and type-2 AGN}

We derive the XLF in relation to the X-ray obscuration. Figure 7 (left panel) shows the TXLF, SXLF, and HXLF (from top to bottom) of X-ray type-1 and type-2 sources. The numbers of sources in each luminosity bin of both types are shown for each energy range (right panel). Table 3 summarises the derived best-fit Schechter parameters. Due to the small number of type- 2 objects, the LFs of type- 1 and type- 2 sources were compared at all redshifts $\mathrm{z} \leq 1.4$, without studying their evolution rates. Furthermore, in order to provide a good Schechter fit of the X-ray type-2 objects in the soft band, the value of the faint-end slope parameter was fixed to -1.5 . Different values were tested, from -1.0 to -2.0 with a bin of 0.25 , and the value of -1.5 was found to give the best fit. In the other two energy ranges the faint-end slope parameter stays free for both type- 1 and type- 2 objects.

The luminosity distributions of X-ray type- 1 and type- 2 objects appear to be similar at both total and hard bands, but significantly different in the soft band, as expected since soft $\mathrm{X}$-rays are especially sensitive to absorption by the central torus.

In general our results suggest that type-1 sources are more numerous at higher luminosities, and type-2 objects dominate the AGN population at lower luminosities. This is known as the 'Steffen effect' (Steffen et al. 2003), and was observed in many later works (e.g., Ueda et al. 2003; Barger et al. 2005; Hasinger et al. 2008). Taking into account uncertainties, the faint-end slopes of $\mathrm{X}$-ray type- 1 and type- 2 sources in the $0.5-7.0 \mathrm{keV}$ and $2.0-10.0 \mathrm{keV}$ bands appear to be similar at the observed redshifts. Moreover, observing the best Schechter-fit parameters, in general type-1 sources show higher characteristic luminosities $\left(L^{*}\right)$ then the type-2 objects. In contrast, the values of the number density $\Phi^{*}$ at characteristic luminosity do not show significant differences between type- 1 and type- 2 sources in any of the three tested $\mathrm{X}$-ray bands.

\subsection{Evolution of X-ray type-1 AGN}

We studied the evolution of X-ray type-1 AGN. Figure 8 shows their TXLF (top panel), as well as the number of sources in each luminosity bin (bottom panel). The evolution rate was studied again in $\mathrm{z}_{1}$ and $\mathrm{z}_{2}$ intervals. Table 4 summarises the best-fit Schechter parameters.

Similarly to the entire AGN sample, evidence for a clear luminosity evolution was found with the most luminous objects residing at higher redshifts. Moreover, we observe a weak evolution in the number densities $\left(\Phi^{*}\right)$, with highredshift X-ray type-1 sources having higher $\Phi^{*}$ values.

\section{Summary}

We present the XLF for X-ray detected AGN in the SXDS and GWS fields. We studied the entire sample of active galaxies as well as different morphological and $\mathrm{X}$-ray subsamples. In the future we will use a much larger sample of X-ray detected AGN from the COSMOS field to derive the LF in relation to the morphology of host galaxies and to study the evolution of different triggering mechanisms (work in progress).

LFs of our entire AGN sample were derived in total, soft, and hard X-ray bands. We studied objects with $\mathrm{z} \leq 1.4$ 
Table 2 The best-fit Schechter parameters of the TXLF as a function of morphology at $\mathrm{z} \leq 1.4$ (Figure 6)

\begin{tabular}{c|c|cccc}
\hline \hline Sample & $\mathbf{N u m}^{a}$ & $\begin{array}{c}\log \Phi^{*} \\
\left(\mathbf{M p c}^{-3}\right)\end{array}$ & $\alpha$ & $\begin{array}{c}\log L^{*} \\
\left.(\mathbf{e r g ~ s})^{-1}\right)\end{array}$ \\
\hline \hline Compact & 79 & $-5.98 \pm 0.84$ & $-1.72 \pm 0.29$ & $44.59 \pm 0.64$ \\
Early-type & 79 & $-6.20 \pm 1.37$ & $-1.98 \pm 0.31$ & $44.46 \pm 0.97$ \\
Late-type & 29 & $-6.63 \pm 3.38$ & $-1.65 \pm 0.57$ & $45.04 \pm 3.68$ \\
\hline \hline
\end{tabular}

${ }^{a}$ Number of objects
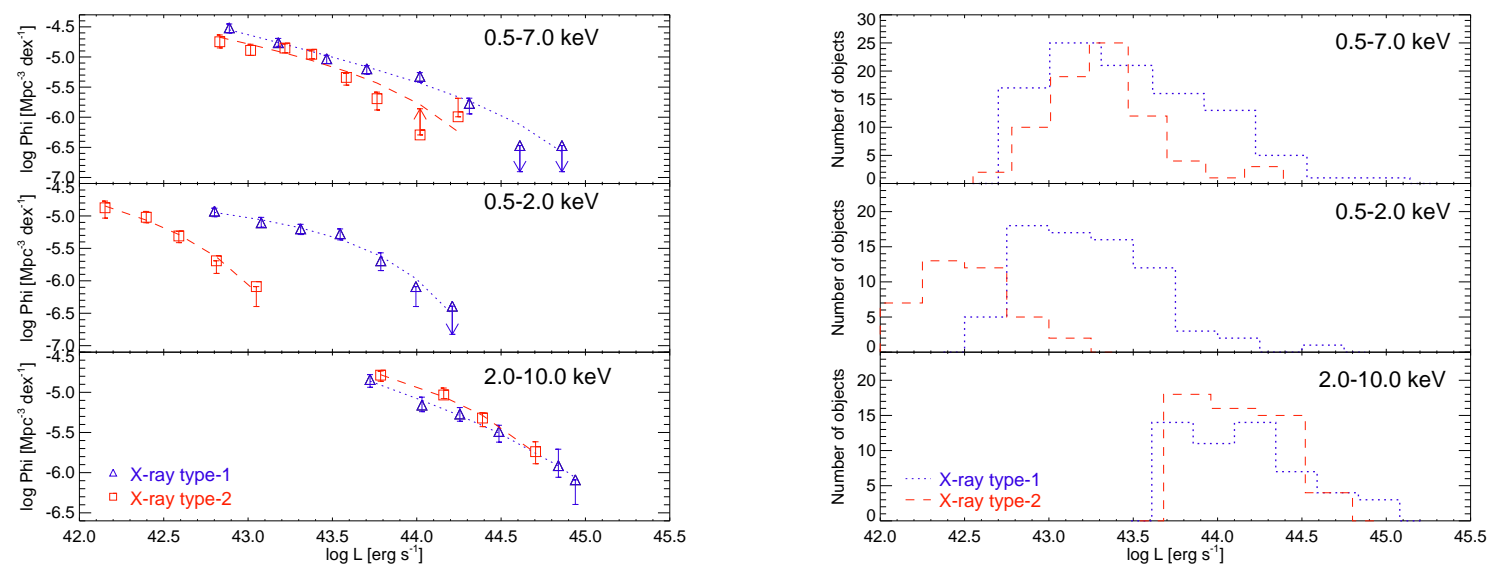

Fig. 7 (Left, from top to bottom): TXLF, SXLF, and HXLF of X-ray type-1 (blue triangles) and type-2 (red squares) objects at redshifts $\mathrm{z} \leq 1.4$. The best-fit Schechter parameters are shown in Table 3 . (Right, from top to bottom): Luminosity distribution in the total, soft, and hard bands of type-1 (blue doted line) and type-2 (red dashed line) sources.

Table 3 The best-fit Schechter parameters of X-ray type-1 and type-2 sources at $\mathrm{z} \leq 1.4$ (Figure 7 )

\begin{tabular}{c|c|cccc}
\hline $\begin{array}{c}\text { En. range } \\
(\mathbf{k e V})\end{array}$ & Sample & Num $^{a}$ & $\begin{array}{c}\log \Phi^{*} \\
\left(\mathbf{M p c}^{-3}\right)\end{array}$ & $\alpha$ & $\begin{array}{c}\log L^{*} \\
\left(\mathbf{e r g ~ s}^{-1}\right)\end{array}$ \\
\hline \hline $\mathbf{0 . 5 - 7 . 0}$ & X-ray type-1 & 120 & $-5.82 \pm 0.47$ & $-1.71 \pm 0.15$ & $44.69 \pm 0.37$ \\
& X-ray type-2 & 92 & $-5.29 \pm 0.47$ & $-1.56 \pm 0.32$ & $43.98 \pm 0.31$ \\
\hline $\mathbf{0 . 5 - 2 . 0}$ & X-ray type-1 & 115 & $-5.13 \pm 0.31$ & $-1.25 \pm 0.3$ & $43.75 \pm 0.23$ \\
& X-ray type-2 & 50 & $-4.99 \pm 0.20$ & $-1.50^{b}$ & $42.68 \pm 0.15$ \\
\hline $\mathbf{2 . 0 - 1 0 . 0}$ & X-ray type-1 & 82 & $-5.75 \pm 0.97$ & $-1.70 \pm 0.40$ & $45.01 \pm 0.72$ \\
& X-ray type-2 & 73 & $-5.05 \pm 0.65$ & $-1.46 \pm 0.55$ & $44.54 \pm 0.45$ \\
\hline \hline
\end{tabular}

${ }^{a}$ Number of objects

${ }^{b}$ Fixed value of $\alpha$ in the Schechter fit

Table 4 The best-fit Schechter parameters of TXLF derived for X-ray type-1 sources (Figure 8)

\begin{tabular}{ccccc}
\hline \hline $\mathbf{z}^{a}$ & $\operatorname{Num}^{b}$ & $\begin{array}{c}\log \Phi^{*} \\
\left(\mathbf{M p c}^{-3}\right)\end{array}$ & $\alpha$ & $\begin{array}{c}\log L^{*} \\
\left(\operatorname{erg~s}^{-1}\right)\end{array}$ \\
\hline \hline $0-0.7$ & 60 & $-4.74 \pm 0.76$ & $-1.37 \pm 0.67$ & $43.66 \pm 0.55$ \\
$0.7-1.4$ & 60 & $-4.28 \pm 0.53$ & $-1.38 \pm 0.45$ & $44.50 \pm 0.37$ \\
\hline \hline
\end{tabular}

${ }^{a}$ Redshift range

${ }^{b}$ Number of objects 


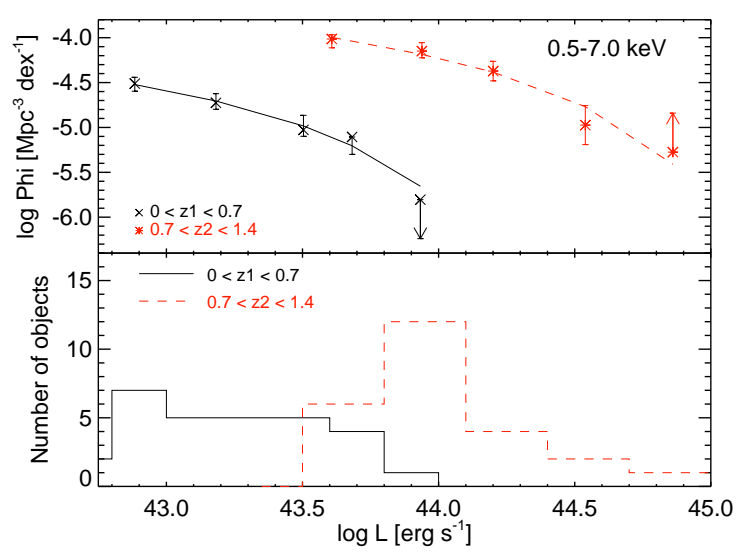

Fig. 8 (Top): TXLF of $\mathrm{X}$-ray type-1 sources derived in two redshift intervals: $\mathrm{z}_{1} \leq 0.7$ (black crosses) and $0.7<\mathrm{z}_{2} \leq 1.4$ (red stars). (Bottom): Distribution of objects in each luminosity bin of $\mathrm{X}$-ray type- 1 sources in the $\mathrm{z}_{1} \leq 0.7$ (solid black line) and $0.7<\mathrm{z}_{2} \leq 1.4$ (dashed red line) redshift intervals. The best-fit Schechter parameters are shown in Table 4.

(where our photometric redshifts are most reliable relative to available spectroscopic redshifts) inferring source evolution in two redshift intervals $\left(0<\mathrm{z}_{1} \leq 0.7\right.$ and $0.7<\mathrm{z}_{2}$ $\leq 1.4$ ) where each redshift band has a similar number of sources. The data were fitted with a Schechter function which was found to be a good approximation in all bands.

The following results were obtained for the XLF derived for the entire sample:

* The LF shape does not change significantly between the two $\mathrm{z}$ intervals for the $\mathrm{X}$-ray bands considered.

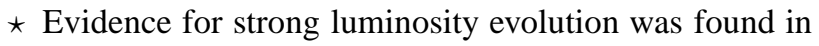
all X-ray bands, with higher density of more luminous objects at higher redshifts.

* Taking uncertainties into account, no significant signs of number density evolution was found in any of the tested bands. Sources become brighter as redshift increases but their characteristic number density $\left(\Phi^{*}\right)$ that corresponds to characteristic luminosity $L^{*}$ appears to remain very much the same in both redshift intervals.

We studied the TXLF as a function of morphology for sources with $\mathrm{z} \leq 1.4$. Three morphological types were tested: compact, early-, and late-type with the following results:

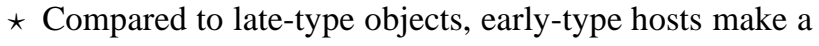
major contribution to the TXLF over the entire luminosity range, while at higher luminosities compact objects become more important.

* No significant differences were found between LFs of compact and early-type objects in the total X-ray band.
The TXLF, SXLF, and HXLF of X-ray type- 1 and type2 sources at $\mathrm{z} \leq 1.4$ were analysed, giving the following results:

* The SXLF of type-1 and type-2 sources are shown to be significantly different due to effects of absorption of soft $\mathrm{X}$-rays by the AGN torus. In the total and hard bands the XLFs remain similar.

* We observed the 'Steffen effect' in this work: type-1 sources are more numerous at higher luminosities, while type- 2 objects are more common at lower luminosities.

* Type-1 sources show higher characteristic luminosities $\left(L^{*}\right)$ than type- 2 objects in the soft and total bands, while at hard energies including uncertainties the properties stay similar.

* Number densities $\Phi^{*}$ at characteristic luminosities do not show significant differences between X-ray type-1 and type- 2 objects in any of three bands.

Finally, the evolutionary rates of X-ray type-1 AGN were studied in $z_{1}$ and $z_{2}$ intervals. Deriving the LFs in each range, we obtained:

* The LFs show similar shape.

* A clear evolution with luminosity was obtained, with more luminous AGN and higher luminosity densities at higher redshifts. We also observed a weak evolution in number densities, with higher redshift sources showing higher $\Phi^{*}$ values.

Acknowledgements. We thank Marc Huertas-Company for a number of valuable comments that improved the morphological classification of analysed sources. We also thank Jack Sulentic and the anonymous referee for all corrections and suggestions. We appreciate all comments from the XMM-Newton team during our SXDS X-ray data reduction, and we also thank the SXDS team for making their data available to the astronomical community. This research has been supported by the Spanish Ministry of Economy and Competitiveness (MINECO) under the grant AYA201129517-C03-01. MP, IM, and JM acknowledge Junta de Andalucía and MINECO through projects PO8-TIC-03531 and AYA201015169. We acknowledge support from the Faculty of the European Space Astronomy Centre (ESAC). JIGS acknowledge financial support from the MINECO under project AYA2008-06311C02-02 and AYA2011-29517-C03-02. JG acknowledges support from the MINECO through AYA2009-10368 project. The CEFCA is funded by the Fondo de Inversiones de Teruel, supported by both the Government of Spain (50\%) and the regional Government of Aragón (50\%). This work has been partially funded by the Spanish Ministerio de Ciencia e Innovaci-ón through the PNAYA, under grants AYA2006-14056 and th-rough the ICTS 2009-14. This research has made use of software provided by the XMM-Newton Science Operations Centre and Chandra X-ray Center (CXC) in the application packages SAS and CIAO, respectively. IRAF is distributed by the National Optical Astronomy Observatories, which are operated by the Association of Universities for Research in Astronomy, Inc., under cooperative agreement with the National Science Foundation. Virtual Observatory Tool for OPerations on Catalogues And Tables (TOPCAT) was used in this work. 


\section{References}

Aird, J., et al., 2010, MNRAS , 401, 2531

Aird, J., et al. 2008, MNRAS, 387, 883

Alexander, D. M., et al. 2003, AJ, 126, 539

Alexander, D. M., et al. 2001, AJ , 122, 2156

Babić, A., et al. 2007, A\&A, 474, 755

Barger, A. J., et al. 2005, AJ, 129, 578

Barger, A. J., \& Hasinger G., 2005, ARA\&A, 43, 827

Bertin, E. \& Arnouts, S., 1996, A\&AS, 117, 393

Blanton, M. R., et al. 2007, AJ 133, 734

Blanton, M. R., et al. 2001, AJ, 121, 2358

Bolzonella, M., Miralles, J.-M., \& Pelló, R 2000, A\&A, 363, 476

Boyle, B. J., et al. 2000, MNRAS, 317, 1014

Boyle, B. J., Shanks, T., \& Peterson, B. A., 1988, MNRAS, 235, 935

Brusa, M., et al. 2009, ApJ, 693, 8

Caccianiga, A., et al. 2004, A\&A, 416, 901

Cepa, J., et al. 2008, A\&A, 490, 1

Cirasuolo, M., Magliocchetti, M., \& Celotti, A. 2005, MNRAS, 357,1267

Civano, F., et al. 2007, A\&A, 476.1223C

Cool, R. J., et al. 2012, ApJ, 748, 10

Cowie, L. L., et al 2003, ApJ, 584, L57

Croom, S. M., et al. 2004, MNRAS, 349, 1397

Croton, D., et al. 2006, MNRAS, 365, 11

Dai, X., et al. 2009, ApJ, 697, 506

Davis, M., et al., 2007, ApJ , 660, 1

Della Ceca, R., et al. 2004, A\&A, 428, 383

De Ruiter, H. R., Arp, H. C., \& Willis, A. G. 1977, A\&AS, 28, 211

Di Matteo T., Springel V., \& Hernquist L. 2005, Nature, 433, 604

Dwelly, T. et al. 2005, MNRAS , 360, 1426

Georgakakis, A., et al. 2009, MNRAS , 397, 623

Ebrero, J., et al. 2009, A\&A, 493, 55

Faber, S. M., et al. 2007, ApJ, 665, 265

Fan, X., et al. 2004, AJ, 128, 515

Fan, X., et al. 2001, AJ, 121, 54

Fanidakis, N., et al. 2012, MNRAS, 419, 2797

Feldmann, R., et al. 2006, MNRAS, 372, 565

Fiore, F., et al. 2012, A\&A, 537, A16

Fiore, F., et al. 2003, A\&A, 409, 79

Furusawa, H., et al. 2008, ApJS, 176, 1

Hasinger, G. et al. 2008, A\&A, 490, 905

Hasinger, G., Miyaji, T., Schmidt, M., 2005, A\&A, 441, 417

Heckman, T. M., et al. 2004, ApJ, 613, 109

Hewett, P. C., et al. 2006, MNRAS, 367, 454

Hodgkin, S. T., et al. 2009, MNRAS, 394, 675

Hogg, D. W. 2000, arXiv: astro-ph/9905116

Hopkins, P.F., et al. 2008, ApJS, 175, 356

Hopkins, P.F., Richards, G. T., \& Hernquist, L. 2007, ApJ, 654, 731

Hopkins, P.F., et al. 2006, ApJS, 163, 1

Huertas-Company, M. et al., 2009, A\&A, 497, 743

Huertas-Company, M. et al., 2008, A\&A, 478, 971

Hunt, M. P., et al. 2004, ApJ, 605, 625

Jiang, L., et al. 2006, AJ, 131, 2788

Kaiser, C. R., \& Best, P. N. 2007, MNRAS, 381, 1548

Kellermann, K. I., et al. 2012, AAS, 21910906

La Franca, F., et al. 2010, ApJ, 718, 368

La Franca, F., et al. 2005, ApJ, 635, 864

La Franca, F., et al. 2002, ApJ, 570, 100

Levenberg, K. 1944, Quarterly of Applied Mathematics, 2, 164

Loveday, J., et al. 1992, ApJ, 390, 338

Mainieri, V. et al. 2002, A\&A, 393, 425
Manners, J. C., et al. 2003, MNRAS, 343, 293

Marquardt, D. 1963, SIAM Journal on Applied Mathematics 11, 431

Marshall, H. L., et al. 1985, ApJ, 299, 109

Matute, I., et al. 2006, A\&A, 451, 443

Merloni, A. 2004, MNRAS, 353, 1035

Miyaji, T., Hasinger, G., \& Schmidt, M. 2001, A\&A, 369, 49

Miyaji, T., et al. 2000, A\&A, 353, 25

Nandra, K., et al. 2005, MNRAS, 356, 568N

Norberg P., et al. 2002, MNRAS, 336, 907

Oke, J. B. \& Gunn, J. E. 1983, ApJ, 266, 713

Ouchi, M., et al. 2008, ApJS, 176, 301

Polletta, M. et al. 2007, ApJ, 663, 81

Page, M. J., \& Carrera, F. J., 2000, MNRAS, 311, 433

Page, M. J., et al. 1997, MNRAS, 291, 324

Perola, G. C., et al. 2004, A\&A, 421, 491

Polletta, M., et al. 2007, ApJ , 663, 81

Pović, M. et al. 2012, A\&A, 541, 118

Pović, M. et al. 2009, ApJ , 706, 810

Press, W. H., \& Schechter, P., 1974, ApJ , 193, 437

Richards, G. T., et al. 2006, AJ, 131, 2766

Richards, G. T., et al. 2005, MNRAS, 360, 839

Schechter, P. 1976, ApJ, 203, 297

Scoville, N., et al., 2007, ApJS, 172, 1

Schmidt, M. et al., 1968, ApJ, 151, 393

Sekiguchi, K. et al., in preparation

Silverman, J. D., et al. 2008a, ApJ , 675, 1025

Silverman, J. D., et al. 2008b, ApJ, 679, 118

Silverman, J. D., et al. 2005, ApJ, 618, 123

Simard, L. 1998, ASPC, 145, 108

Sheth, R. K., \& Tormen, G., 1999, MNRAS , 308, 119

Steffen, A. T., et al. 2003, ApJ, 596, L23

Sutherland, W., \& Saunders, W. 1992, MNRAS , 259, 413

Tody, D., 1993, Astronomical Data Analysis Software and Systems II, A.S.P. Conference Ser., Vol 52, eds. R.J. Hanisch, R.J.V. Brissenden, \& J. Barnes, 173

Tody, D., 1986, Proc. SPIE Instrumentation in Astronomy VI, ed. D.L. Crawford, 627, 733

Trenti, M., Stiavelli, M., 2008, ApJ , 676, 767

Ueda, Y., et al., 2003, ApJ , 598, 886

Warren, S. J., et al. 2007, MNRAS , 375, 213

Wu, Y., et al. 2011, ApJ, 734, 40

Yencho, B., et al. 2009, ApJ , 698, 380

Younes, G., et al. 2012, A\&A, 539, 104 